\title{
Calibration of the Mass-Luminosity Relation Using Trigonometric Parallaxes. I.
}

\author{
WILLIAM F. VAN ALTENA, TERRENCE M. GIRARD, \& \\ JOHN T. LEE \\ Yale Astronomy Department, P.O. Box 6666, New Haven, CT 06511, USA
}

\begin{abstract}
The calibration of luminosities using trigonometric parallaxes introduces well-known errors that are a function of the ratio of the parallax error to the parallax, when the sample is chosen from stars with measured parallaxes larger than some minimum parallax. In this paper it is shown that similar errors are also introduced into the mass axis of the mass-luminosity relation (MLR) and can result in a biased MLR. The bias is shown to be related to the Lutz-Kelker correction to the absolute magnitude, as extended by Hanson for the case of selection effects in the data sample. The size of the correction in the mass axis is substantial and for the case of a uniform distribution in space, it can amount to a multiplicative factor of 1.17 in the mass for a ten sigma $(\sigma / \pi=0.10)$ parallax and a factor of 1.62 for a five sigma $(\sigma / \pi=0.20)$ parallax.
\end{abstract}

\section{INTRODUCTION}

It has been known for many years that the direct use of trigonometric parallaxes to calibrate the luminosities of stars can result in values biased towards lower luminosities, when the sample is chosen from stars with measured parallaxes larger than some minimum parallax. The origin of this error is in the rapidly increasing number of stars encountered per unit parallax as one moves to larger and larger distances. When this parallax distribution, $\mathrm{G}(\pi)$, is convolved with an observational error, the result is that many more stars with small true parallaxes are scattered towards larger observed parallaxes than conversely. As a result, an uncorrected calibration uses parallaxes that are on the average too large, which results in a luminosity or absolute magnitude that is too dim. A good example of this systematic error is given by Hoffleit (1939), where she compared the trigonometric and spectroscopic parallaxes for a sample of 370 bright southern stars with spectroscopic parallaxes on the Mt. Wilson system. Hoffleit plotted the differences between the absolute magnitudes determined from the trigonometric and spectroscopic parallaxes versus the trigonometric parallaxes. For the small and consequently poorly measured parallaxes, she showed that there were large systematic errors in the derived absolute magnitudes.

The first paper that dealt in detail with the problem was that of Dyson (1926), in which he derived the formalism for correcting the observed distribution of parallaxes to the true distribution using Eddington's (1913) method. The true distribution could then be used to derive a luminosity calibration without systematic error. The method was subsequently described by Trumpler \& Weaver (1953) and Feast \& Shuttleworth (1965). Wallerstein (1967) dealt with the problem in the calibration of the $\mathrm{K}$-line absolute magnitudes through Monte Carlo simulations of a sample of stars. The statistical corrections thus estimated were used to correct the absolute magnitudes derived from the observed trigonometric parallaxes. Following this, Lutz \& Kelker (1973) formalized the 
problem and analytically calculated the corrections to be applied to the derived absolute magnitudes for a uniform distribution of stars in space. Hanson (1979) then generalized the problem by devising a method to determine the true spatial distribution of the observed stars and then calculated the correction appropriate for that spatial distribution. The latter effect is very important, since various selection effects introduce spatially non-uniform distributions. The revised corrections can sometimes be as much as a factor of two smaller than the original Lutz-Kelker corrections.

\section{THE ABSOLUTE MAGNITUDE CORRECTIONS}

In this section we will review the basics of the Lutz-Kelker corrections in order to relate them to the corrections to the mass calibration. We will follow the formalism developed by Lutz \& Kelker (1973) and Hanson (1979). Given the distance modulus equation, we can write the error in the absolute magnitude, $\Delta M=M_{t}-M_{o}$, where the subscripts $t$ and $o$ refer to the true and observed quantities, as

$$
\Delta M=5 \log \left(\pi_{t} / \pi_{0}\right) .
$$

For simplicity, we will henceforth drop the $t$ subscript and assume that unsubscripted quantities refer to the true values. If we have a uniform distribution of stars in space, then the number of stars between $r$ and $r+d r$ is given by,

$$
N(r) d r=4 \Pi D_{\circ} r^{2} d r,
$$

where $D_{\circ}$ is the star density and $I=3.14159$. The equivalent number of stars between $\pi$ and $\pi+d \pi$ is,

$$
N(\pi) d \pi=4 \Pi D_{\circ} \pi^{-4} d \pi .
$$

Equation 3 is the parallax distribution for a uniform distribution of stars in space. Hanson's (1979) modification of the distribution assumes that the distribution goes as $\pi^{-n}$, where $n$ is to be determined rather than assumed to be equal to 4 . We therefore have,

$$
N(\pi) d \pi=4 \Pi D_{\circ} \pi^{-n} d \pi .
$$

It remains now to determine the exponent, $n$, in the density law so that the correction can be evaluated. Hanson (1979) pointed out that since the parallax and the proper motion are directly related by $\pi=4.74 \mu / V$, and the kinematic distribution of stars is relatively independent of spatial location in the solar neighborhood, we can write $z=\pi / \pi_{0} \propto \mu / \mu_{0}$. For most of the stellar samples of interest, the rms velocity is about $35 \mathrm{~km} \mathrm{~s}^{-1}$. As a result, the proper motion errors are some eight times smaller in proportion to the proper motion, than the parallax errors are to the parallax. The proper motion errors can therefore be safely ignored as an important contributor to the smearing of the true proper motion distribution in the region of interest. The distribution of the proper motions can then be approximated by,

$$
g(\mu) \propto \mu^{-n},
$$


and the cumulative proper motion distribution is then,

$$
N(\mu)=\int_{\mu_{0}}^{\infty} g(\mu) d \mu \propto \mu^{-(n-1)},
$$

or taking logs,

$$
\log N(\mu)=-(n-1) \log \mu+\text { const. }
$$

Hanson (1979) has shown that for samples of stars known to be complete, an index of $n=4$ is obtained, in agreement with the expected value for a uniform distribution in space. Values as small as $n=2.5$ have been found for samples with a strong proper motion bias such as for the subdwarfs used to calibrate the Pop II main sequence. The result of this small value of $n$ is that smaller LutzKelker corrections, by about a factor of two, are derived and a larger value of the limiting value of $(\sigma / \pi)_{\max }$ can be used, thus allowing us to use parallaxes with somewhat larger errors and practically speaking, more stars. To differentiate the corrections, $\Delta M(n)$, for an arbitrary power law distribution in space (or selection effect) from those with a uniform distribution $(n=4)$, we will refer to the former as the Lutz-Kelker-Hanson or LKH corrections.

\section{DEFINITION OF THE CALIBRATION SAMPLE}

Some care must be exercised in the definition of the sample that we are going to calibrate. For example, the LK corrections are applicable strictly to a sample selected with $\pi \geq \pi_{1}$, where $\pi_{1}$ is the lower observed parallax selection limit. If the sample has been selected such that, in addition $\mu \geq \mu_{1}$, then the LKH corrections should be used after determining the appropriate value of the power law in the spatial distribution, i.e. $n$ in Equation 7. If the sample satisfies the above criteria, then the formalism in the above section applies.

\section{THE MASS CORRECTIONS}

Turning now to the mass axis, we note that the quantity determined is the sum of the masses, $\sum m=\left(m_{1}+m_{2}\right)$, which is given by,

$$
\sum m=a^{3} P^{-2} \pi^{-3},
$$

and

$$
\begin{aligned}
& m_{2}=F \sum m ; \\
& m_{1}=(1-F) \sum m,
\end{aligned}
$$

where $F=m_{2} / \sum m$ is the fractional mass as determined from photographic astrometry. Since we normally deal with the masses in logarithmic units, we take the $\log$ of Equation 9 and get,

$$
\log m_{2}=\log F+3 \log a-2 \log P-3 \log \pi .
$$

The systematic error introduced into the masses will then be given by, 


$$
\Delta \log m=\log m_{t}-\log m_{o},
$$

or

$$
\Delta \log m=-3 \log \left(\pi / \pi_{\circ}\right),
$$

where $m_{t}$ and $m_{0}$ are the true and observed masses, respectively. Recall that the error in the magnitude axis was given by Equation 1 as

$$
\Delta M=5 \log \left(\pi / \pi_{0}\right) \text {. }
$$

The similarity of Equation 12 and Equation 13 show that we can write the correction to the mass axis as,

$$
\Delta \log m=-(3 / 5) \Delta M_{L K H} .
$$

As an example of the new correction to the mass axis, we have computed the corrections to both axes in the Mass-Luminosity relationship for binary stars with calculated orbits in the new edition of the Yale Parallax Catalogue (YPC). However, we have not yet included the mass ratios for the stars and must assume that each binary consists of two main sequence stars. Binaries with YPC spectral type information indicating a non-dwarf component are excluded from this sample. In Paper II we will provide more details on the calculations, analyze all binaries in the YPC and include the mass ratios in the discussion.

We turn now to estimating the true distribution of the binaries in space in order to determine the value of $n$ to be used in Equation 4. In Figure 1, we plot $\log N(\mu)$ versus $\log \mu$ for those binaries with measured parallaxes $\pi_{\circ} \geq+0.030^{\prime \prime}, \geq+0.040^{\prime \prime}$ and $\geq+0.050^{\prime \prime}$ and $(\sigma / \pi) \leq 0.20$. The slope of the "linear" part of the relation provides an estimate of the spatial distribution power law index, $n$. The values have been estimated by eye and yield $n=2.8$, 3.0 , and 2.9 , respectively for the above minimum parallaxes. We adopt $n=2.9$ as a value representative of binaries that might be appropriate to use for the calibration.

We should emphasize that the space distribution of the binaries in our sample is the product of many selection effects. If, for example, we attempt to trace the various selection effects, we must first discover the binary nature of the star, which in general imposes a maximum apparent magnitude of about 9.0. While fainter binaries can be discovered today, the existence of an observational history adequate to determine an orbit implies that the binary was discovered long ago with a relatively small telescope. And second, some parallax observer must have thought that it was close enough to the sun to warrant spending the time to determine its parallax. The separate characterization of these selection effects would be both difficult and suspect, so the use of Hanson's (1979) approach to estimate the space distribution power law index of the sample will be adopted.

In Table 1 below we have used a power law index of $n=2.9$ determined from Figure 1 to illustrate the range of the corrections to be applied to the two axes. The low value of $n$ enables the calibration to extend to stars with $(\sigma / \pi) \leq$ 0.287 , rather than the more restrictive value of 0.175 for the $n=4$ case. The details of the restriction to the maximum value of $(\sigma / \pi)$ are discussed in detail by Hanson (1979). In Table 1 the columns list the ratio of the parallax error to the parallax, the LKH correction to the absolute magnitude, the systematic 


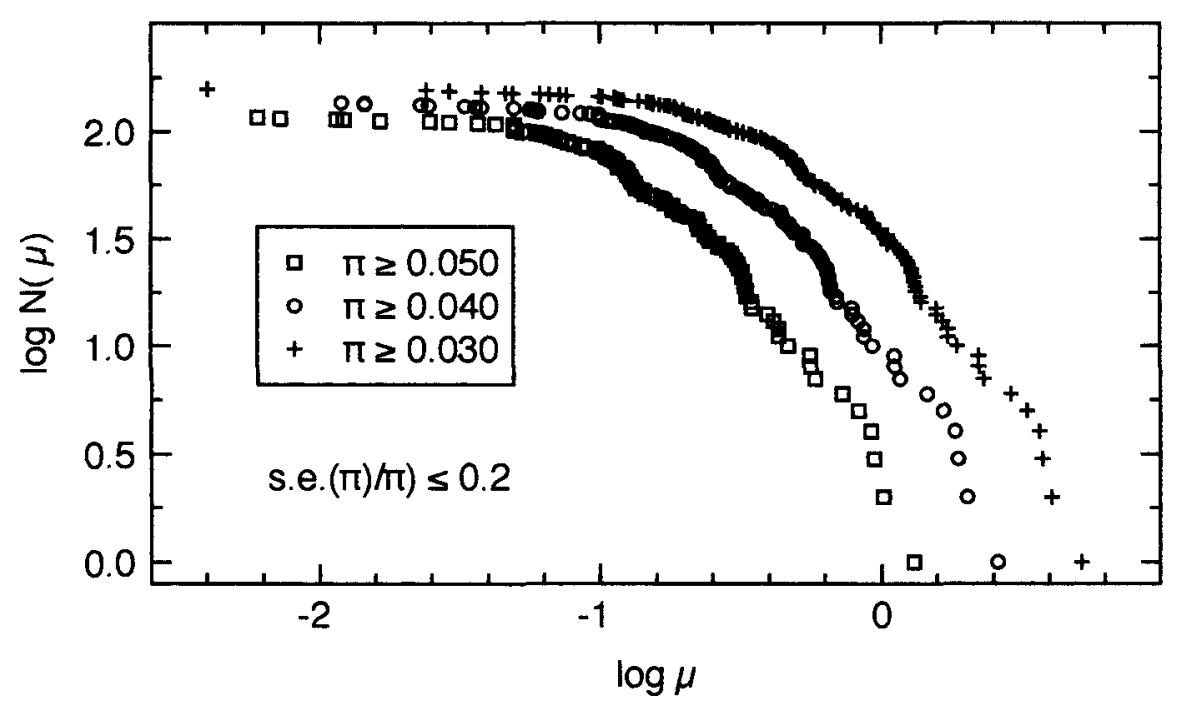

FIGURE 1. The log of the cumulative distribution versus the $\log$ of the proper motion for $\pi \geq+0^{\prime \prime} .030,+0^{\prime \prime} .040$, and $+0^{\prime \prime} .050$ and $(\sigma / \pi) \leq 0.020$. The proper motions have been scaled by factors of two to offset the curves for clarity.

correction to the log of the mass, and the ratio of the true (corrected) mass to the observed mass.

It is clear from Table 1 that the LKH corrections to the absolute magnitudes are very large at the point, $(\sigma / \pi)=0.3$, where the derivation of a correction is vitiated by a variety of other errors that will dominate the final values of the bolometric magnitude and the $\log$ of the mass. Note that for $(\sigma / \pi) \geq 0.20$, the values of the corrections depend strongly on the integration limits for the individual star and may therefore differ from those listed in Table 1, which are values averaged over several stars with approximately the same $(\sigma / \pi)$.

TABLE 1. Systematic corrections to the masses and luminosities for a parallax power law distribution index $\boldsymbol{n}=\mathbf{2 . 9}$.

\begin{tabular}{|cccc|}
\hline$\sigma / \pi$ & $\Delta M_{L K H}$ & $\Delta \log m$ & $m / m_{0}$ \\
\hline 0.00 & 0.00 & 0.000 & 1.00 \\
0.05 & -0.02 & 0.012 & 1.03 \\
0.10 & -0.08 & 0.048 & 1.17 \\
0.15 & -0.19 & 0.114 & 1.30 \\
0.20 & -0.35 & 0.21 & 1.62 \\
0.25 & -0.50 & 0.30 & 2.00 \\
0.30 & -0.65 & 0.39 & 2.45 \\
\hline
\end{tabular}




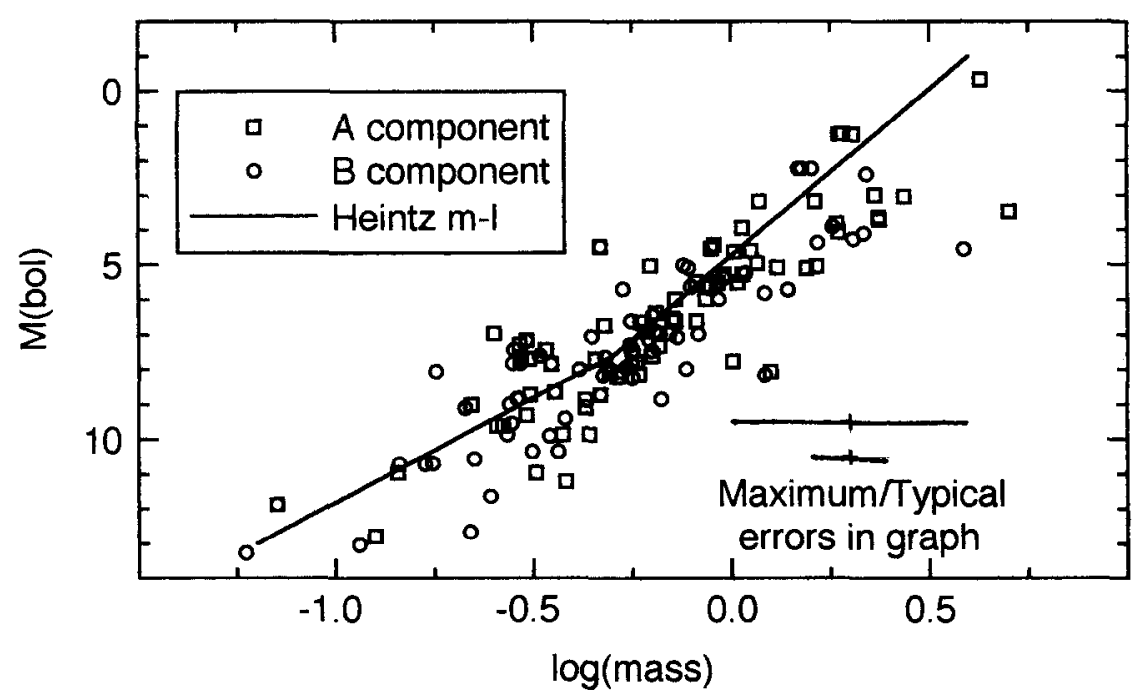

FIGURE 2. Mass-Luminosity Relation for stars with errors $\leq 0.3 \mathrm{mag}$ and $\log$ (mass), respectively, corrected as described in the text for systematic errors in both axes. The pairs of stars are assumed to lie parallel to the ZAMS in the color-magnitude diagram to deconvolve them into individual visual magnitudes and colors, and they are subsequently assumed to lie parallel to the ZAMS in the MLR to determine the unknown mass fraction.

\section{CALIBRATING THE MASS-LUMINOSITY RELATION}

It has been recognized for many years that accidental errors in the measured parallaxes introduce a systematic error into absolute magnitude calibrations. It has therefore become a more or less standard practice to apply the Lutz-Kelker corrections to the absolute magnitudes of the stars before deriving the calibration. Corrections to the mass have not been applied before since this is the first time that their existence has been discussed as far as we are aware. One would therefore expect that the commonly used calibrations published by Harris, Strand and Worley $(1963)$ and Heintz $(1978,1983)$ would be changed significantly by the new corrections. As illustrated in Figure 2 the relations are altered only slightly for two reasons. First, the massive end of the Mass-Luminosity Relation is dominated by masses determined from spectroscopic and eclipsing binaries, which are independent of trigonometric parallaxes and therefore the apparent deviation of the observations from the line is not significant; and second, the low mass end is dominated by well determined parallaxes, which therefore have relatively small systematic corrections. The location of the break in the slope of the Mass-Luminosity relation near $M_{b o l}=+7$ and the details of the distribution of the stars around the relation may, however, be distorted by the neglect of the corrections.

We note only briefly here that there appear to be systematic deviations for 
the low mass stars and that the MLR may be in need of adjustments due to the application of the new corrections to the masses. We will defer further analysis of the data to Paper II after we have included all of the available data and the mass ratios in the calculations.

\section{CONCLUSIONS}

The procedures used to calibrate the Mass-Luminosity relation have been reexamined in relation to the systematic errors introduced into the two coordinates by the accidental observational errors in the trigonometric parallaxes. It is shown that the now standard LKH corrections to the bolometric magnitude axis have analogous systematic corrections that must be applied to the mass axis. The size of the corrections in the mass axis are substantial and in this case can amount to a multiplicative factor of 1.17 in the mass for a ten sigma $(\sigma / \pi=0.10)$ parallax and a factor of 1.62 for a five sigma $(\sigma / \pi=0.20)$ parallax.

\section{ACKNOWLEDGMENTS}

It is a pleasure to acknowledge numerous conversations over the years with Drs. R. B. Hanson and T. E. Lutz concerning the importance of recognizing and dealing with systematic errors in, and caused by, observational errors in trigonometric parallaxes. van Altena would like to thank the Director of the Department of Astronomy and Meterorology of the University of Barcelona and Dr. Jorge Nunez for providing facilities and hospitality while this paper was being written. This research was supported in part by grants from NASA, NSF and the DGICYT of Spain under project SAB91-0232.

\section{REFERENCES}

Dyson, F. 1926, MNRAS, 86, 686

Eddington, A. 1913, MNRAS, 73, 359

Feast, M.W. \& Shuttleworth, M. 1965, MNRAS, 50, 244

Hanson, R.B. 1979, MNRAS, 186, 875

Harris, D.L., Strand, K.Aa., \& Worley, C.E. 1963, in Basic Astronomical Data edited by K. Aa. Strand, (Chicago, University of Chicago Press), p. 273

Heintz, W.D. 1978, Double Stars, (Dordrecht, D. Reidel Publ. Co.), p. 59

Heintz, W.D. 1983, Current Techniques in Double and Multiple Star Research, IAU Colloquium 62 edited by R. S. Harrington and O.G. Franz, Flagstaff, Arizona p. 10

Hoffleit, D. 1939, The Astron. Obs. of Harvard College, Bull. 911, 24

Lutz, T.E. \& Kelker, D.H. 1973, PASP, 85, 573

Lutz, T.E.. 1979, MNRAS, 189, 273

Trumpler, R.J. \& Weaver, H.F. 1953, Statistical Astronomy, (Berkeley, University of California Press) p. 369

Wallerstein, G. 1967, PASP, 79, 317 


\section{DISCUSSION}

HARRINGTON: Have you done $\log (\mathrm{N}(\mathrm{M})) / \log \mu$ plots as a function of spectral type to see how the slope changes and thus where incompleteness sets in as you go down the main sequence?

VAN ALTENA: No, but this is one of the things we will do once we have completed our data base.

MAZEH: Can you comment on the slopes of the lines of the figure.

VAN ALTENA: The slopes of the lines in the mass-luminosity relation are those from the 1978 book of W. D. Heintz.

HEINTZ: The slope of the lower main sequence was revised from 6.0 to about 6.5 - nothing very significant.

ABT: In the mass-luminosity diagram, the upper line does not seem to be drawn through the mean position of the points.

VAN ALTENA: The upper line is defined by eclipsing binaries, not the visual binaries that are illustrated here.

KROUPA: On the lower main sequence, uncertainties in bolometric correction are very large.

VAN ALTENA: Yes, the bolometric corrections must contribute a significant error to the bolometric magnitudes. 